\title{
The possibility of separation of electronic waste by means of their electrical properties
}

\author{
Mikolaj Skowron ${ }^{1, a}$, Agnieszka Surowiak ${ }^{1}$, Przemyslaw Syrek ${ }^{1}$, Antoni Ciesla ${ }^{1}$, Wojciech Kraszewski ${ }^{1}$, and Damian \\ Lachendrowicz ${ }^{1}$
}

${ }^{1}$ AGH University of Science and Technology, al. Mickiewicza 30, 30-059 Krakow, Poland

\begin{abstract}
Electric field has a lot of applications in technology. One of them is electrodynamic separation: electric field influences selectively granular solids of different moments or charges. A mathematical model of the separation process in high voltage drum separator is presented in the paper. Particles are charged both by induction and corona phenomena: next, they are separated by effects of the field forces. Some computational and experimental results are given and analyses.
\end{abstract}

\section{Introduction}

Each year, in the European Union around 8 million tons of waste of electrical and electronic equipment (the annual growth rate of formation of these wastes is $3-5 \%$ all produced), while worldwide, there are annually around 20-50 million tons of waste. One of the objectives of rational resource consumption and waste is the best use of the primary components of natural resource and recovery largely processed elements from waste products. The processing of various types of waste for recovery of useful components can be used by separation methods in mineral processing. Then the separation of useful chemical elements of the waste is carried out for separation of well-defined characteristics. This attribute, which is called the argument of the chapter, could be the difference in the densities of particle components to be separated, settling velocity of particles, wettability (hydrophobic and hydrophilic), magnetic susceptibility, electrical conductivity and other $[1-8]$.

The electromagnetic field is used for different of technological processes (separation of magnetic and electrodynamic $[9,10]$ ), medicine (magnetic therapy, diagnostics) $[11,12]$. Electrodynamic separation is the process of separating two or more components with different physical properties, the forces of the electric field. One group of separation processes is represented by the conductance methods. These methods are based on different electric conductance of separated particles, which lead a various time constants of their charging. The applications of electrodynamic separation technologies range from recovery of metallic and insulating materials from industrial wastes [13 - 16], mineral beneficiation [17 - 19] and metals recovery from electronic scarp [20 22].
The paper presents how high voltage drum separator works. The analysis of electric field distribution in separator working space was done. Additionally, distribution of forces acting on particles of dielectric and conductive properties was examined, trajectories of charged particles movement were presented as well. The laboratory investigations of electronic wastes were performed, and in such a process the particle of size $0-0.5 \mathrm{~mm}$ was obtained. It was observed that as a result of selective charging of particles they separate according to surface ability to electrifying what allows to obtain selective separation of components being so-called electronic wastes. The application of electrodynamic drum separator allows to separate such chemical elements as $\mathrm{Ti}, \mathrm{Cu}, \mathrm{Fe}, \mathrm{Pb}, \mathrm{Sn}$ from plastics occurring in electronic wastes.

The motion of dielectric particles in electric field is described by the formula:

$$
\boldsymbol{F}_{e l}=\boldsymbol{F}_{d}+\boldsymbol{F}_{e}=\boldsymbol{\mu} \nabla \boldsymbol{E}+Q \boldsymbol{E}
$$

where: $\boldsymbol{F}_{e l}$ is the electric field force, $\boldsymbol{F}_{d}$ denotes its dielectrophoresis component, $\boldsymbol{F}_{e}$ stands for its electrophoresis component, $\boldsymbol{\mu}$ is the electric moment of the particle, $\boldsymbol{E}$ represents the electric field, and $Q$ denotes the electric charge of the particle.

The involved forces of and principal methods of charging dielectric particles are schematically shown in Fig. 1.

\footnotetext{
${ }^{a}$ Corresponding author: mskowron $@$ agh.edu.pl
} 


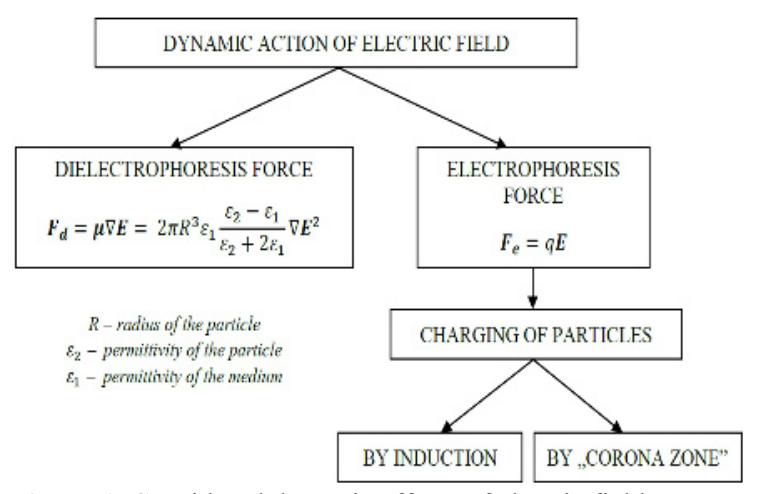

Figure 1. Considered dynamic effects of electric field.

The prevailing structure in mineral processing (beneficiation) technology is the „corona” drum separator invented by Huff [23] (Fig. 2).

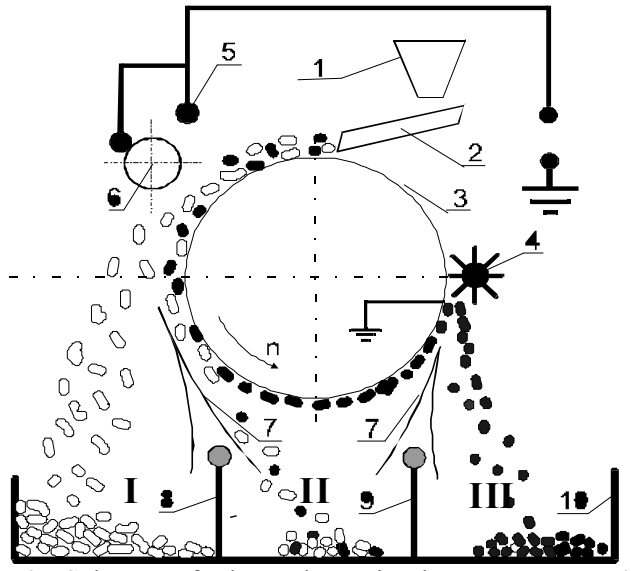

Figure 2. Scheme of electrodynamic drum separator (Huff's separator):

1- feed container, 2-feed hopper, 3-drum, 4-brush, 5-corona electrode, 6-deflecting electrode, 7-separation barriers, 8, 9, 10bins for separation products.

\section{Mathematical model of electric field dynamics in the drum separator}

The mixture of grains falls out of the hopper $(\boldsymbol{E}=0)$ into direct field ( $\boldsymbol{E}=$ const.) generated between two cylindrical electrodes (one of them rotates). The particles in contact with the rotating electrode obtain an electric charge by induction. The electrical scheme for the ,particle - drum electrode" system is presented in Fig. 3. The dependence of the acquired charge on time is defined by the formula [24]:

$$
\begin{aligned}
& Q(t)=U \cdot \frac{C_{p} C_{z}}{C_{p}+C_{z}}+U \cdot \frac{C_{p}^{2}}{C_{p}+C_{z}} \\
& {\left[1-\exp \left(\frac{-t}{R_{z}\left(C_{p}+C_{z}\right)}\right)-Q_{0} \cdot \exp \left(\frac{-t}{R_{z}\left(C_{p}+C_{z}\right)}\right)\right]}
\end{aligned}
$$

where: $Q_{0}$ is the charge of the particle acquired in corona zone given by the expression

$$
Q_{0}=\left(1+2 \frac{\varepsilon_{2}-\varepsilon_{1}}{\varepsilon_{2}+\varepsilon_{1}}\right) \varepsilon_{0} E \pi r^{2}
$$

here $\varepsilon_{2}$ is the relative permittivity of the particle, $\varepsilon_{1}$ denotes the relative permittivity of the ambient medium and $r$ stands for the radius of the particle. The other parameters $C_{p}, C_{z}$ and $R_{z}$ follow from Fig. 3 .

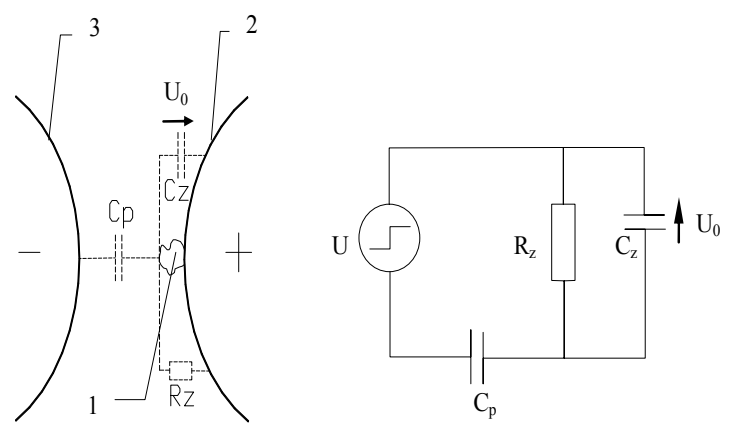

Figure 3. Particle on rotating drum in an electrodynamic drum separator (the sketch and the scheme):

1 - particle, 2 - rotating drum, 3 - counter electrode

The examples of dependences of acquired charges on time are shown in Fig. 4.

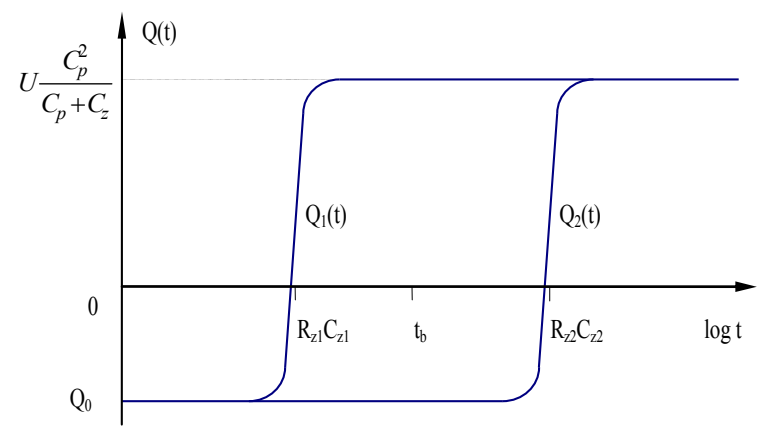

Figure 4. Particle charges vs. time in drum separator for $U_{0} \neq 0$.

The analysis leads to the conclusion that the separation takes place when the contact time $t_{b}$ between the mixture and drum is longer than the time constant of the ,conductor" grain, but shorter than the time constant for the „nonconductor" particle (particle of the „conductor”, e.g. the component , 1 " $-z_{1}$; particle of the ,nonconductor", e.g. the component ,2" $2-z_{2}$ ). In other words, the separation takes place if

$$
R_{z 1} C_{z 1}<<t_{b}<<R_{z 2} C_{z 2}
$$

A particle, charged according to formula (2), is passing through electric field between two cylindrical electrodes (Fig. 2). The equation for the forces influencing a particle has the form:

$$
m \frac{d v}{d t}=\Sigma \boldsymbol{F}
$$

where: $m$ is the mass of the particle, $v$ denotes its velocity, and $\Sigma \boldsymbol{F}$ stands for the sum of forces acting on the particle. 
Both particles on the surface of the rotating drum and particles passing through the electric field are affected by forces of the following modules:

$$
\begin{array}{ll}
\text { - electric field force } & F_{\mathrm{e}}=Q E \\
\text { - , image” force } & F_{\mathrm{im}}=\frac{Q^{2}}{4 \pi \varepsilon_{0}\left(2 R_{2}^{2}\right)} \\
\text { - centrifugal force } & F_{\mathrm{c}}=m \omega^{2} R_{2} \\
\text { - gravitational force } & F_{\mathrm{g}}=m g \\
\text { - aerodynamic resistance } & F_{\mathrm{a}}=6 \pi \mu_{d} r v
\end{array}
$$

where $R_{2}$ is the radius of the rotating drum, $\omega$ denotes the angular velocity of rotation, $g$ stands for the acceleration by gravity, and $\mu_{\mathrm{d}}$ is the coefficient of the dynamic viscosity of the ambient medium.

The scheme of forces influencing a particle during the contact with the rotating drum as well as during the following fall is presented in Fig. 5.

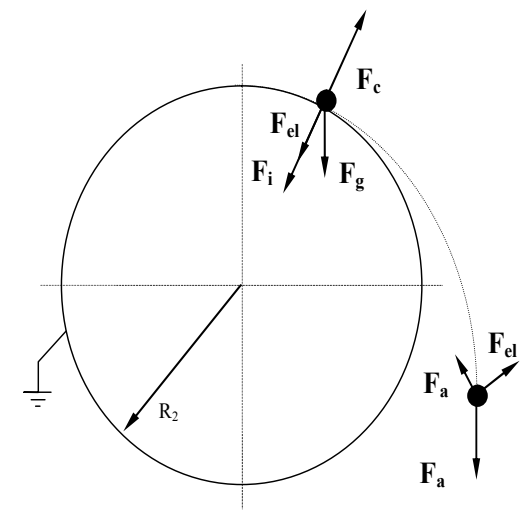

Figure 5. Scheme of forces influencing particle in drum separator.

The trajectories of particles in the separators were calculated on the basis of the field distribution (formula (5) as well as the forces influencing the particles (formulae (6)-(10)). For calculations, the software package „Matlab" was used. The results of the calculations (several trajectories) for various dynamic conditions are presented in Fig. 6.

a)

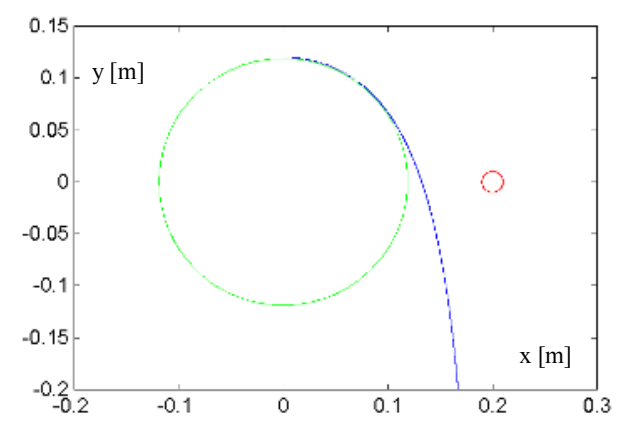

b)

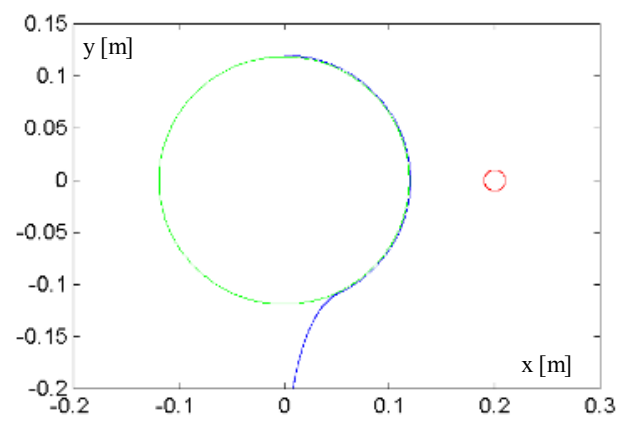

c)

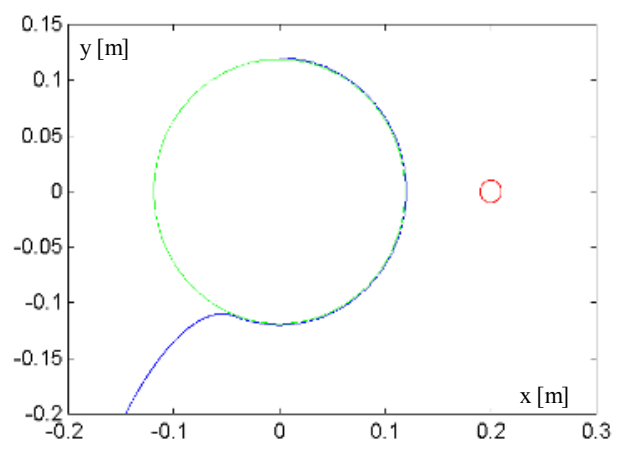

Figure 6. Exemplary particle trajectories (computer simulation): a) $R_{z}=1 \mathrm{E} 6[\Omega]$, b) $R_{z}=4 \mathrm{E} 6[\Omega]$, c) $R_{z}=7 \mathrm{E} 6[\Omega], U=20[\mathrm{kV}] ; \omega$ $=8\left[\mathrm{rads}^{-1}\right]$

\section{Experimental investigation}

\subsection{Research methodology}

The high-voltage electrodynamic drum separator consists of two functional parts, namely of the charging part and of the electrophoretic separation part Fig.7.

The key problem of the separation - selective charging of the mixture components - can be based on various physical phenomena. From the practical point of view, these phenomena are electrostatic induction and occurrence of the corona charge. The prevalent construction in mineral processing (beneficiation) technology is the corona drum separator invented by Huff [24]. A scheme of this separator is presented in Fig. 2.

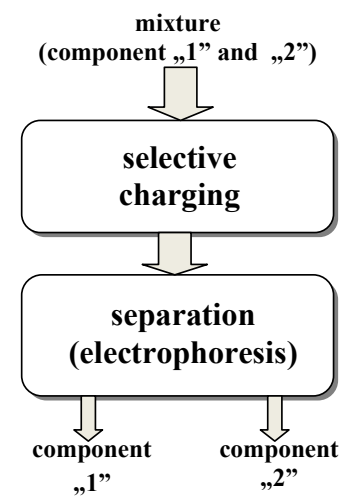

Figure 7. Electrodynamic separation based on the electrophoresis phenomenon 
A mixture is poured out of the hopper and passes through the corona zone (the particles assume to have negative charges). Next, the mixture falls on the rotating drum. The particles of small resistivity (consequently, of small relaxation times) lose their charges rapidly. Subsequently, they take up some charges of opposite sign and are repelled by the field forces into the bin I. However, particles of large relaxation time (of great resistances) lose little of no charge: they are ,pinned" to the rotating drum by means of the ,image" forces. Eventually, the particles are removed with the brush into the bin III. The intermediate product falls into the bin II and may be returned to the feed hopper. A theoretical model of the process was presented by the authors [25].

The device presented above may be analyses as a control object - carrying into effect electro separation as a technological process (Fig. 8). The state of the process is defined by state variables $\mathrm{X}(t)$. The object can be controlled by a number of input values, called control values $\mathrm{U}(t)$. Other input values, so called disturbances $\mathrm{Z}(t)$, are undesirable for the control process.

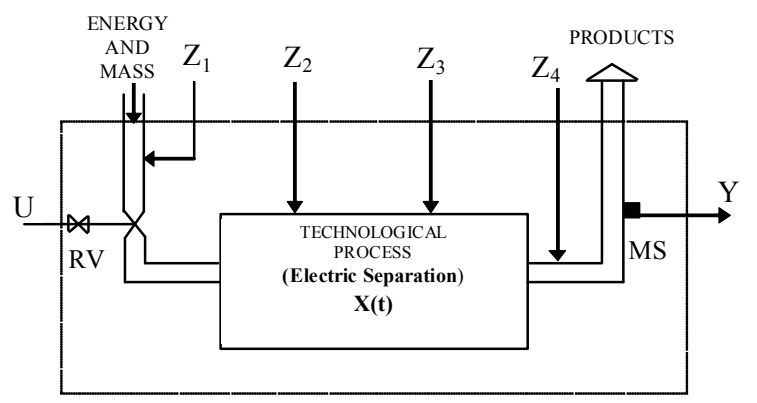

OBJECT OF CONTROL

Figure 8. Technological process (electric separation) as an object of control: X, U, Y- vectors of variables of state, control and outlet (respectively), $Z_{1} \ldots Z_{4}$ vectors of disturbances, $R V-$ regulation valves, MS - measurement sensors

The key problem of the separation - selective charging of the mixture components - can be based on various physical phenomena. From the practical viewpoint, these phenomena are electrostatic induction and occurrence of the corona charge. The prevalent construction in mineral processing (beneficiation) technology is the corona drum separator invented by Huff. A scheme of this separator is presented in Fig. 2.

A mixture is poured out of the hopper and passes through the corona zone (the particles assume to have negative charges). Next, the mixture falls on the rotating drum. The particles of small resistivity (consequently, of small relaxation times) lose their charges rapidly. Subsequently, they take up some charges of opposite sign and are repelled by the field forces into the bin I. However, particles of large relaxation time (of great resistances) lose little of no charge: they are ,pinned" to the rotating drum by means of the ,image” forces. Eventually, the particles are removed with the brush into the bin III. The intermediate product falls into the bin II and may be returned to the feed hopper. A theoretical model of the process was presented by the authors [25].
The device presented above may be analyzed as a control object - carrying into effect electro separation as a technological process (Fig. 8). The state of the process is defined by state variables $\mathrm{X}(t)$. The object can be controlled by a number of input values, called control values $\mathrm{U}(t)$. Other input values, so called disturbances $\mathrm{Z}(t)$, are undesirable for the control process.

The most important input values for the separation process are the voltage $V$, geometry of the electrodes (their distance $D$ ), rotational speed of the drum $\omega$, angle of inclination of the corona electrode $\alpha$, and concentration of the tested component in the feed $\beta$. The disturbances are, for example, the temperature $T$, pressure $p$ or moisture $\delta$. The state variables are the particle charge $Q$, electric field $\boldsymbol{E}$, and granulation of the feed $d$. The separation process described above results in a product of flow off $\gamma$ and tested component concentration $\lambda$, which are the quantities suitable for the evaluation of quality of the separation process.

\subsection{Research station}

Electrodynamic separation was performed using a laboratory drum separator (Fig. 9). On the basis of the available literature [3], separation and numerical calculations and series of simulations, the separator is selected with the following parameters: the voltage of the electrodes $11 \mathrm{kV}$ and the rotation of the drum $60 \mathrm{rev} / \mathrm{min}$

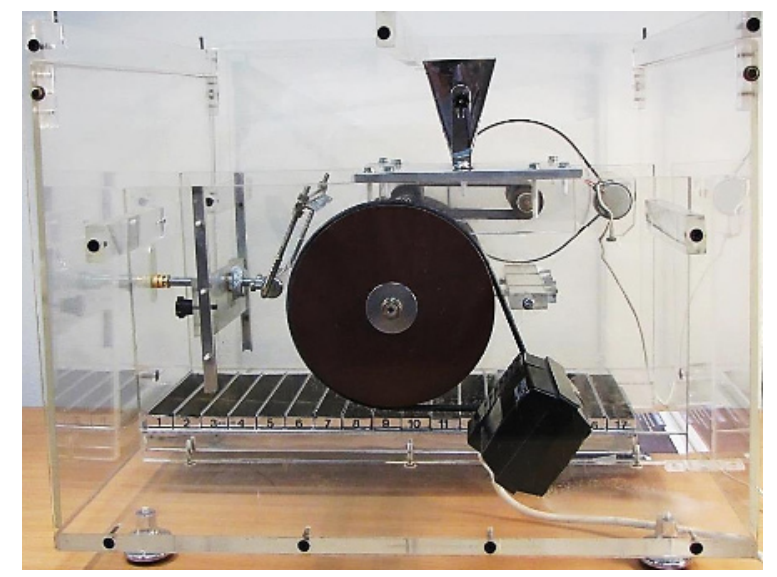

Figure 9. Photo electrodynamic drum separator

\subsection{Research of separation of electronic wastes}

The material directed to separation was electronic wastes from computer mainboards. Firstly, they wer comminuted in knife crusher and the material of granulation $<0.5 \mathrm{~mm}$ was sieved. Such prepared sample was separated in electrodynamic drum separator by keeping constant temperature and humidity conditions. The products of separation were collected in containers numbered from 1 till 10. The material from each container was weighted and mass shares of separation products were calculated. In each of products collected in individual containers 
the contents of the following elements were marked: Ti, Fe, $\mathrm{Cu}, \mathrm{Sn}, \mathrm{Pb}, \mathrm{Bi}$. They were presented in Table 1 .

The distributions of chosen elements ( $\mathrm{Ti}, \mathrm{Cu}, \mathrm{Fu}, \mathrm{Pb}, \mathrm{Sn}$ ) in separated material which gathered in containers numbered from 1 till 10 after separation conducted in electrodynamic drum separator were presented on Figs. $10-15$.

Table 1. Contents of individual elements in products of electronic wastes separation

\begin{tabular}{|c|c|c|c|c|c|c|c|}
\hline $\begin{array}{c}\text { Nr of } \\
\text { conteiner }\end{array}$ & $\begin{array}{c}\text { Mass } \\
{[\%]}\end{array}$ & $\begin{array}{c}\mathrm{Ti} \\
{[\%]}\end{array}$ & $\begin{array}{c}\mathrm{Fe} \\
{[\%]}\end{array}$ & $\begin{array}{c}\mathrm{Cu} \\
{[\%]}\end{array}$ & $\begin{array}{c}\mathrm{Sn} \\
{[\%]}\end{array}$ & $\begin{array}{c}\mathrm{Pb} \\
{[\%]}\end{array}$ & $\begin{array}{c}\mathrm{Bi} \\
{[\%]}\end{array}$ \\
\hline 1 & 5.4 & 0.7 & 7.2 & 73.2 & 12.3 & 13.2 & 0.0 \\
\hline 2 & 5.4 & 0.8 & 8.2 & 66.8 & 10.7 & 11.1 & 0.0 \\
\hline 3 & 4.3 & 0.8 & 10.3 & 57.5 & 9.3 & 9.9 & 0.1 \\
\hline 4 & 7.3 & 1.1 & 11.2 & 36.1 & 5.1 & 5.5 & 1.1 \\
\hline 5 & 6.5 & 1.5 & 8.8 & 28.6 & 3.7 & 4.5 & 1.0 \\
\hline 6 & 7.0 & 1.6 & 4.9 & 20.7 & 2.1 & 2.6 & 1.2 \\
\hline 7 & 4.8 & 1.2 & 7.3 & 13.8 & 2.6 & 2.9 & 1.3 \\
\hline 8 & 3.8 & 2.2 & 6.3 & 9.7 & 1.4 & 2.0 & 1.7 \\
\hline 9 & 10.8 & 1.7 & 5.2 & 3.3 & 0.6 & 1.0 & 1.8 \\
\hline 10 & 44.9 & 0.0 & 4.7 & 2.9 & 1.1 & 1.2 & 1.0 \\
\hline
\end{tabular}

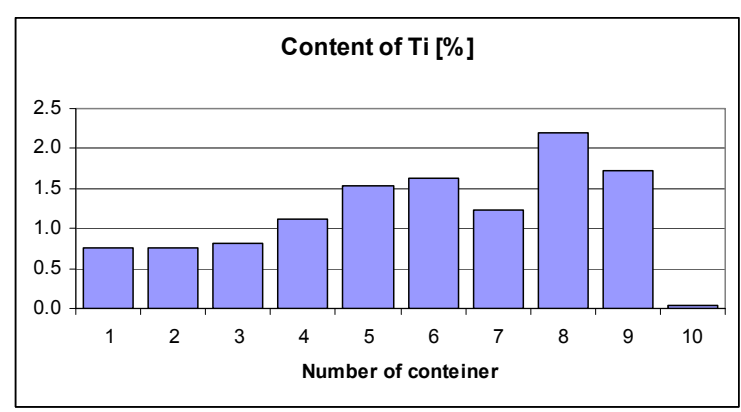

Figure 10. Distribution of Ti contents in separation products

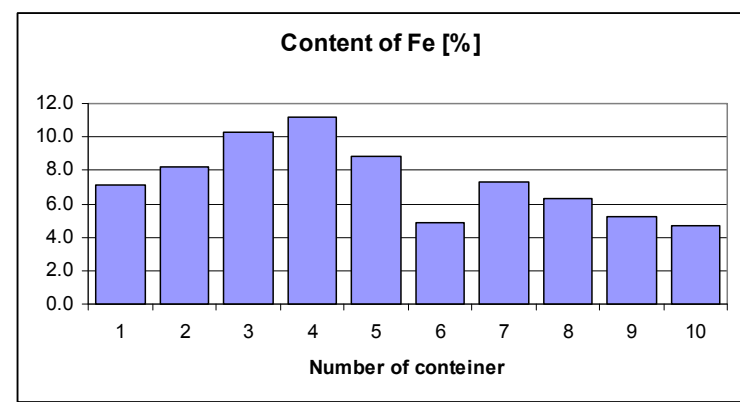

Figure 11. Distribution of Fe contents in separation products

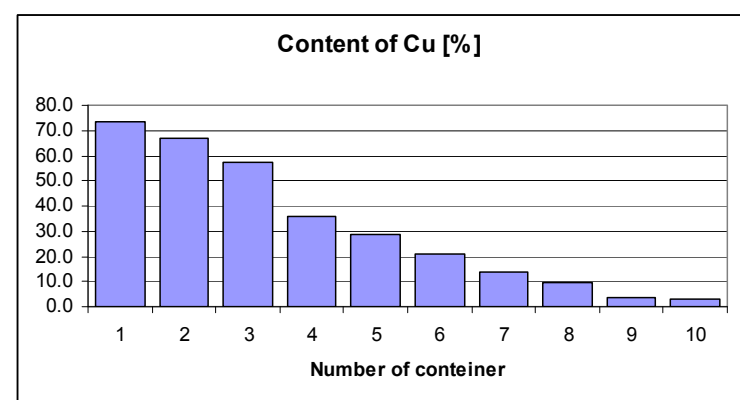

Figure 12. Distribution of $\mathrm{Cu}$ contents in separation products

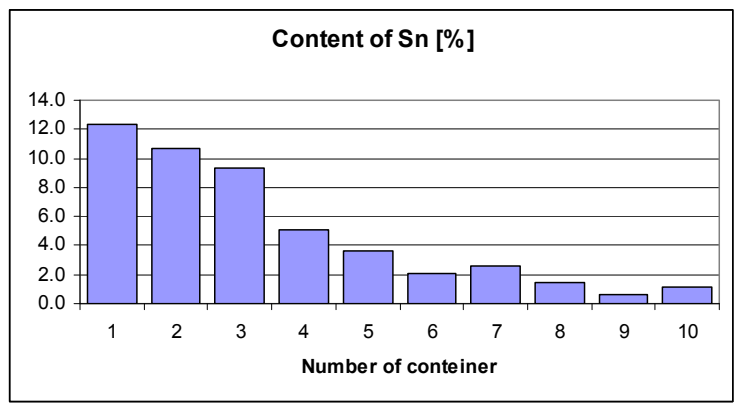

Figure 13. Distribution of Sn contents in separation products

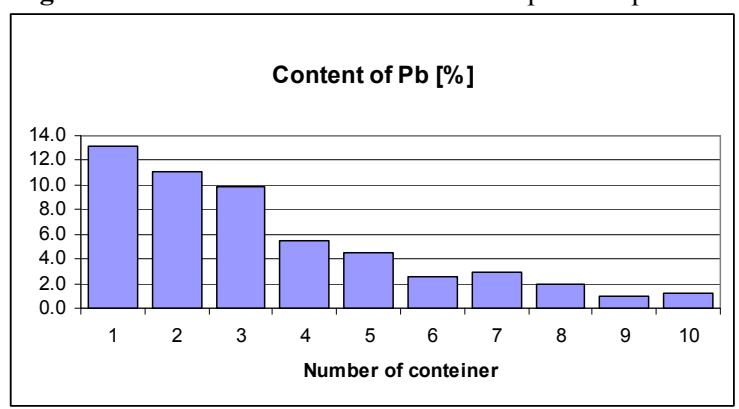

Figure 14. Distribution of $\mathrm{Pb}$ contents in separation products

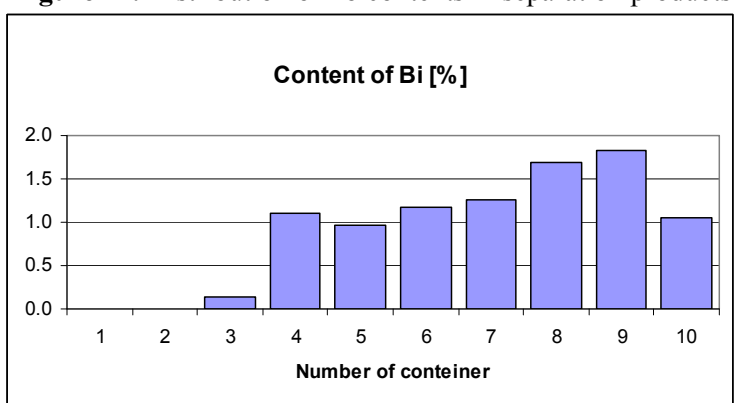

Figure 15. Distribution of Bi contents in separation products

Analysis of the results shows that the titanium content is concentrated in containers numbered from 4 till 9 and it can be seen that quite high levels of $\mathrm{Cu}$ and $\mathrm{Sn}$ occur in containers with lower numbers (1-3). Distribution of iron contents in containers is rather irregular. The maximum Fe content can be obtained from containers of numbers 3 and 5 while $\mathrm{Bi}$ concentrates in containers of numbers 4 till 10 . Such a distribution of the elements contents is associated with the particle size distribution of the material, which influences on degree of the elements liberation as well possibility of the surface electrification.

\section{Summary}

Both theoretical analyses and experiments confirmed the usefulness of electrodynamic drum separators to separation of mixed granular solids. The required condition is that separated materials must characterize with different resistivity. The experiments proved that behavior of particles (treated as control objects) is mainly related to the electric field and rotary speed of the drum. Any unambiguous interpretation of the results is difficult because a lot of mechanical and electrical factors act simultaneously. 
Analysis of the results leads to the following conclusions and observations:

- during electrodynamic separation the conductive components can be separated from the non-conductive electronic waste. In addition, the decomposition of some elements can be observed during the process. It can be concluded that the separation of $\mathrm{Ti}$ components from the rest is successful. Products containing such elements as $\mathrm{Cu}, \mathrm{Pb}$ and $\mathrm{Sn}$ should be separated by means of another method in purpose of separating these elements. Various metals can be recovered in processes of metallurgy or hydrometallurgy. Products containing iron should be directed to purification by means of magnetic separation.

- advantage of electrostatic separation is that it is conducted in dry conditions. Therefore, there are no additional processes of thickening and dewatering, what eliminates the necessity of additional devices.

\section{References}

1. Brozek M., Surowiak A., Physicochem. Prob. of Min. Proc. 41, 397-413 (2007).

2. Brożek M., Surowiak A., Jarosiński A., Arch.of Metal. and Mat., 60(3), 1737-1744 (2015).

3. Ciesla A., Kraszewski W., Skowron M., Surowiak A., Syrek P., Min. Res. Manag. 31(1), 155 - 174 (2016).

4. Niedoba T. Physicochemical Problems of Mineral Processing, 49(1), 175-188, (2013).

5. Surowiak A., Mineral Resources Management, V. 30, Iss. 1, pp. 105-122, (2014).

6. Surowiak A., Brożek, M.,. Arch. of Minin. Sci., 59(1), 269-282 (2014a).

7. Surowiak A., Brożek M., Arch. of Minin. Sci., 59(2), 553-562 (2014b).

8. Surowiak A., Brożek M., Physicochem. Prob. of Min. Proc. 52(1), 228-243 (2016).

9..Ciesla A., Przegl. Elektrotech., 87(7),: 99-103, (2011).

10. Ciesla A., Przegl. Elektrotech., 88(12B), 50-53, (2012).

11. Ciesla A., Kraszewski W., Skowron M., Syrek P., Przegl. Elektrotech, 87, 79-82 (2011).

12. Ciesla, A., Syrek, P., 88(12B), 124-127 (2012).

13. Lawver E., Dyrenforth W.P., Electrostatics and its Applications, (Wiley, New York, 1973).

14. Chong-Qing Wang, Hui Wang, You-Nian Liu, Waste Manag., 35, 42-47 (2015).

15. Kachi, M., Dascalescu, L., Herous, L., Nemamcha, M., Industry Applications, IEEE Trans., 49(2), 691 - 698 (2013).

16. Wu Jiang, Li Jia, Xu Zhen-Ming, J. Hazard. Mater. 161, 257-262 (2009).

17. Bittner J. D., Flynn K. P., Hrach F. J. Intern. Min. Proc. Cong., Chile, (2014).

18. Cui J., Forssberg E., J. of Hazar. Mat. 99(3), 243-263 (2003).

19. Lockhart N.C., Powder Techn., 40(1-3), 17-42, (1984).

20. Birloaga I., De Michelis I., Ferella F., Buzatu M., Veglio F., Waste Manag., 33, 935-941 (2013).

21 Veit H.M., Diehl T.R., Salami A.P., Rodrigues J.S., Bernardes A.M., Tenorio J.A.S., Waste Manag., 25, 67-74, (2005).
22. Li J., Lu H., Liu S., Xu Z., J. of Hazard. Mat. 153, 269275 (2008).

23. Ralston O. C., Electrostatic Separation of Mixed Granular Solids. (Amsterdam - New York, 1961).

24. Szczerbiński M., J. of Electrostat, 175 - 186, (1983).

25. Cieśla A., Proceedings ISH'99, London, (1999). 